\title{
A Laplace transfer-based citation model and its application in detecting the obsolescence characteristics of articles
}

\author{
Mingyang Wang ${ }^{1, *}$, Dongtian Leng ${ }^{1}$, Yang $\mathrm{Li}^{1}$ and Xiangrong Zhang ${ }^{2, *}$ \\ College of Information and Computer Engineering, Northeast Forestry University, Harbin 150040, People's Republic of China \\ College of Economics and Business Administration, Heilongjiang Institute of Technology, Harbin 150050, People's Republic of China
}

\begin{abstract}
This study describes a Laplace transfer-based citation model proposed for the actual citation process among journals. The actual citation process is a dynamic physical system which includes two continuous processes of ageing and publishing of cited articles. By deducing the Laplace transfer function for these two processes, we have established the input-output function for the actual citation process. Considering the subject of 'automation and control systems' as an example, time parameters associated with the ageing process were identified from the input-output function to examine the obsolescence characteristics for the subject. Experimental results show that the ageing time parameter calculated by the Laplace transferbased citation model is a good expression on the obsolescence of the field. The field shows a moderate ageing rate and a shortened obsolescence tendency on the whole. Most of the articles cited annually in this field are articles published 5-10 years ago. There is a monotonic decreasing dependence between the increment of IF and the increment of time parameter $T_{1}$, although IF always increases regardless of whether the ageing tends to prolong or shorten.
\end{abstract}

Keywords: Citation process, dynamic physical system, impact factor, journal articles, obsolescence.

OBSOLESCENCE of articles is usually considered as the decrease in the validity of information with time; it is also known as literature ageing. In order to measure the speed and degree of scientific literature obsolescence, and reveal obsolescence law quantitatively, researchers have put forward some main measures such as cited half-life ${ }^{1-5}$, price index ${ }^{6}$, prospective price index ${ }^{7}$, and the other useful indicators from different perspectives ${ }^{8-14}$. Obsolescence from both the synchronous and diachronous perspectives has been widely discussed in the literature ${ }^{15-18}$.

In fact, obsolescence of scientific literature is not only an objective social phenomenon, but also a complex dynamic process. The observed citing process of periodicals includes the dissemination process of cited papers,

\footnotetext{
*For correspondence. (e-mail: wmynefu@163.com;
}

xiangrongzh@163.com) the ageing process of cited papers, and the publishing process of citing papers ${ }^{19}$. That is to say, when an article $A$ is cited by an article $B$, the delay of citation actually involves the dissemination process and the ageing process of article $A$, and the publication process of article $B$. However, we are accustomed to calculating the citation age of one cited article from its publication time till the publication of the citing article; publication delays thus exist in the citation distribution of the cited article. Egghe and Rousseau ${ }^{19}$ proposed a research-citation cycle to examine the role of publication delay on the citation of an article. Yu and co-workers proposed a physical model to simulate the research-citation cycle and deduced the expression of periodical average publication delay ${ }^{20-23}$. Based on these works, this article describes a Laplace transfer-based citation model proposed for the actual citation process and the universal input-output function deduced for it. By taking the universal input-output function to simulate the actual cumulative citation distribution, the time parameters associated with the ageing process were identified to describe the obsolescence of journal articles. Taking the field of 'automation and control systems' as an example, we discuss the application of this Laplace transfer-based citation model on examining the obsolescence characteristics of articles.

\section{Method}

Egghe and Rousseau ${ }^{19}$ have reported that the actual citation process of articles includes three steps as mentioned earlier. As the citation activities among journals or subjects are actually based on the mutual citation relationship between micro-articles, the actual citation process among journals or the higher aggregated level of subjects should also include these three processes of dissemination of the cited periodical (subject) articles, ageing of the cited periodical (subject) articles and publishing of the citing periodical (subject) articles. Figure 1 shows a sketch for the actual citation process among journals (subjects).

The dissemination process of articles refers to the procedure by which they enter into the bibliographic repository and can be retrieved by the readers. The process has 


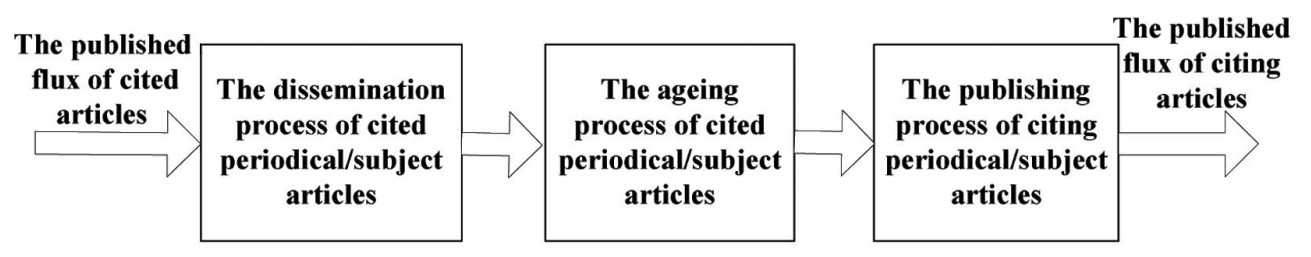

Figure 1. Sketch for the actual citation process among journals/subjects.

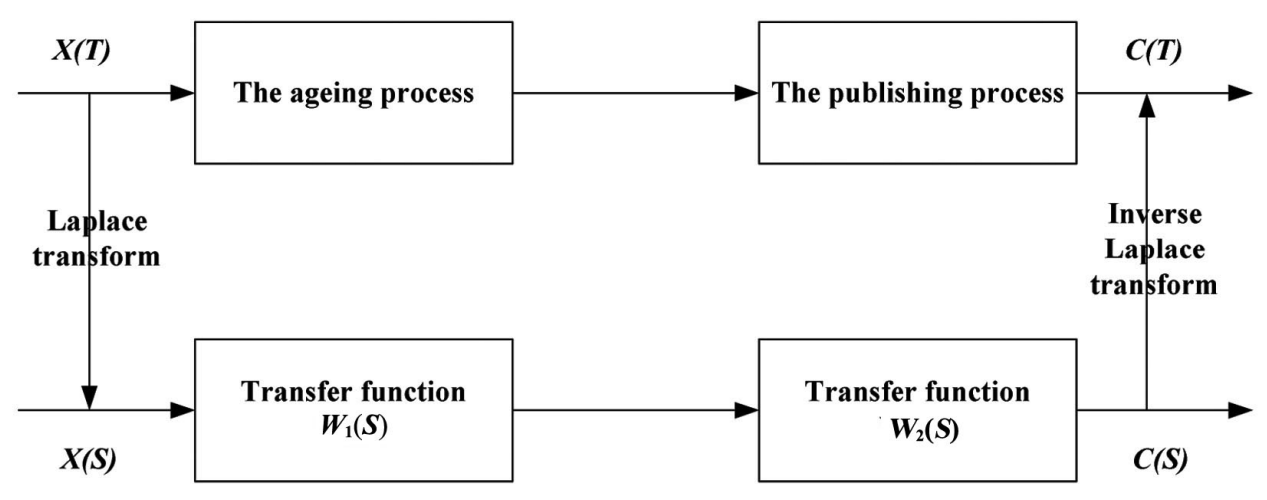

Figure 2. Laplace transfer-based citation model.

been greatly reduced with the application of internet and database technologies, and so it can be ignored throughout the total citation lifecycle of articles. The ageing process refers to the procedure by which the content of scientific literature becomes obsolete with its 'time of life' increasingly, as the value of the literature decreases simultaneously. This ageing process will be reflected in the citation distribution of the cited articles. The publication process gives the publication delay for citing articles between submission and publication. This delay is also hidden in the citation distribution of the cited articles.

Laplace transform is a fundamental tool for analysing variable systems, which has been widely used in various linear or nonlinear systems ${ }^{24-26}$. The actual citation process can be regarded as a physical system; the output of the system changes with the change in the input. Based on the actual citation process shown in Figure 1, a Laplace transfer-based citation model has been proposed for the actual citation process as shown in Figure 2, where $X(T)$ is the input of the system, $S$ the Laplace operator, $X(S)$ the Laplace transform of the input $X(T) . C(T)$ the output of the system, and $C(S)$ is the Laplace transform of the output $C(T) . W_{1}(S)$ is the transfer function model of the ageing process of cited articles and $W_{2}(S)$ is the transfer function model of the publishing process of citing articles.

The input $X(T)$ of this transfer function model is the total citation rate to one cited journal (subject) $A$, and

$$
X(T)= \begin{cases}1, & T \geq 0, \\ 0, & T<0 .\end{cases}
$$

$T$ refers to the age of articles in journal (subject) $A$. The Laplace transfer of input $X(T)$ is

$$
X(S)=\frac{1}{S}
$$

The articles in journal (subject) $A$ are systematically exported through the actual citation process, including their own ageing process and the publishing process of the citing articles.

\section{Transfer function of the ageing process}

The classical Bernal negative exponential model describes the ageing of the literature, as shown in the following equation

$$
f(T)=K \cdot \exp (-\alpha T),
$$

where $T$ is the age of the literature, $K$ a constant and $\alpha$ is the aging coefficient.

Based on eq. (3), the cumulative citation distribution function can be obtained

$$
C(T)=1-\mathrm{e}^{-\alpha T},
$$

$C(T)$ is the output function of $X(T)$, that is a step response. Suppose $T_{1}$ is the ageing time parameter in this process, and $T_{1}=1 / \alpha$. Based on the Laplace transform theory, the transfer function $W_{1}(S)$ of the ageing process should be

$$
W_{1}(S)=\frac{1}{T_{1 S}+1} .
$$




\section{Transfer function model of the publishing process}

$\mathrm{Yu}$ and co-workers have proposed the continuous model for dynamic periodical publishing process and deduced a group of partial differential equations to express publishing process at the steady state ${ }^{20-23}$

$$
\left\{\begin{array}{l}
\frac{\mathrm{d} N / t}{\mathrm{~d} t}=X(t)-Y(t), \\
N(t)=\int_{\tau}^{\infty}(T, t) \mathrm{d} T, \\
Y(t)=\int_{\tau}^{\infty} y(T, t) \mathrm{d} T \\
\frac{\partial n(T, t)}{\partial T}+\frac{\partial n(T, t)}{\partial t}=-y(T, t), \\
y(T, t)=P(T, t) \cdot n(T, t), \\
n(\tau, t)=X(t), \\
N(0)=N_{0}, \\
n(T, 0)=n_{0}(T), \\
X(0)=X_{0}, \\
Y(0)=Y_{0},
\end{array}\right.
$$

where $T$ is the literature age, $t$ a time variable, $X(t)$ the accepted contribution flux of publications, $\tau$ the pure time delay caused by the review process, $N(t)$ the deposited contribution quantity, $n(T, t)$ the age distribution of the deposited contribution, $Y(t)$ the published literature flux, $Y(T, t)$ the age distribution of the published literature flux and $P(T, t)$ is the age distribution of the literature publishing probability.

At the steady state, the various parameters for a periodical will tend to a dynamic equilibrium state after some time. The adopted contribution flux is a constant and is equal to the published literature flux. The deposited contribution quantity is also a constant. All the factors do not change with time, and so we have $n(T, t)=n(t), y(T$, $t)=y(T), P(T, t)=P(T)$. Then the model of periodical publication delay at steady state is

$$
\left\{\begin{array}{l}
X=Y, \\
N=\int_{\tau}^{\infty} n(T) \mathrm{d} T, \\
Y=\int_{\tau}^{\infty} y(T) \mathrm{d} T, \\
\frac{\mathrm{d} n(T)}{\mathrm{d} T}=-y(T), \\
y(T)=P(T) \cdot n(T), \\
\text { The boundary condition: } n(\tau)=X .
\end{array}\right.
$$

Supposing the literature is published with equal probability, then we have

$$
\begin{aligned}
& n(T)=X * \mathrm{e}^{-P(T)(T-\tau)}, \\
& y(T)=P(T) * X^{*} \mathrm{e}^{-P(T)(T-\tau)},
\end{aligned}
$$

Then

$$
\begin{aligned}
& N=\int_{0}^{\infty} n(T) \mathrm{d} T=\int_{0}^{\infty} X * \mathrm{e}^{-P(T-\tau)} \mathrm{d} T=\frac{X}{P}, \\
& y(T)=\frac{X^{2}}{N} * \exp \left(-\frac{T-\tau}{N / X}\right) .
\end{aligned}
$$

Let $T_{S}=N / Y$, we obtain

$$
y(T)=\frac{Y}{T_{S}} * \exp \left(-\frac{T-\tau}{T_{S}}\right)
$$

The cumulative distribution function of publishing process can be obtained by integral operation of eq. (10) in the delay interval of $[0, T]$

$$
\int_{0}^{T} \frac{y(T) \mathrm{d} T}{Y}=1-\exp \left(-\frac{T-\tau}{T_{s}}\right)
$$

Suppose $G(T)=\int_{0}^{T}(y(T) \mathrm{d} T) / Y$ we can get the cumulative probability distribution function of the publishing process

$$
G(T)=1-\exp \left(-\frac{T-\tau}{T_{s}}\right)
$$

Then the transfer function $W_{2}(S)$ could be obtained by the Laplace transfer of eq. (12)

$$
W_{2}(S)=\frac{\mathrm{e}^{-\tau s}}{T_{s}(s+1)} \text {. }
$$

Combining the Laplace transfer functions of $W_{1}(S)$ and $W_{2}(S)$, the universal Laplace transfer function of the actual citation process can be obtained

$$
W(s)=W_{1}(S) \cdot W_{2}(S)=\frac{\mathrm{e}^{-\tau s}}{\left(T_{1}(s+1)\right)\left(T_{S}(s+1)\right)} .
$$

The Laplace transfer $C(S)$ of output $C(T)$ can be easily deduced based on $X(S)$ and $W(S)$.

$$
C(S)=W(S) \cdot X(S)=\frac{\mathrm{e}^{\tau s}}{\left(T_{1}(s+1)\right)\left(T_{S}(s+1) s\right)} .
$$


Using the inverse Laplace transform of $C(S)$, the cumulative citation rate distribution function $C(T)$ of journal (subject) $A$ is obtained

$$
C(T)=1-\frac{T_{1} \cdot \exp \left(-\frac{T-\tau}{T_{1}}\right)}{T_{1}-T_{S}}+\frac{T_{S} \cdot \exp \left(\frac{T-\tau}{T_{S}}\right)}{T_{1}-T_{S}},
$$

where $T_{1}$ is the ageing time parameter related to the obsolescence of articles in journal (subject) $A$, which refers to the average ageing rate of all articles in it $A$ which were cited in one collection year. $T_{S}$ is the publishing time constant related to the average publication delay of citing articles. $\tau$ is the publication pure delay related to the review delay of citing articles.

Based on the cumulative citation rate distribution function $C(T)$ in eq. (16) and the actual cumulative citation distribution of one journal (subject), we can easily calculate the three time parameters. The time parameter, $T_{1}$, identified from the ageing process, can be used to characterize the obsolescence of articles.

From $C(T)$ in eq. (16), the formula of the cited half-life can also be deduced. Let $C\left(T_{0.5}\right)=0.5$; then the cited halflife $T_{0.5}$ would satisfy the following expression

$$
1-\frac{T_{1} \cdot \exp \left(-\frac{T_{0.5}-\tau}{T_{1}}\right)}{T_{1}-T_{S}}+\frac{T_{S} \cdot \exp \left(-\frac{T_{0.5}-\tau}{T_{S}}\right)}{T_{1}-T_{S}}=0.5 .
$$

Equation (17) can be rewritten as follows

$$
T_{1} \cdot \exp \left(-\frac{T_{0.5}-\tau}{T_{1}}\right)-T_{S} \cdot \exp \left(-\frac{T_{0.5}-\tau}{T_{S}}\right)=0.5 \cdot\left(T_{1}-T_{S}\right) .
$$

Thus, by establishing the Laplace transfer model on the actual citation process among journals (subjects), we can not only identify the ageing time parameter of $T_{1}$, but also the ageing parameter of the cited half-life. As shown in the calculation process, all the citations have been incorporated when calculating $T_{1}$, which makes it more accurate than the cited half-life to describe obsolescence characteristics of the journals. In this study, the time parameter of $T_{1}$ was mainly used to characterize the obsolescence of 'automation and control systems'.

\section{Data}

'Automation and control systems' is a rapidly growing, interdisciplinary field of science that applies methods from mathematics, information science and computer science to solve problems in automatic control science. Analysing the citation patterns could help researchers un- derstand how it has evolved as a dynamic interdisciplinary field. Practising information professionals or librarians also could utilize the detected citation patterns for understanding the users' needs and developing the collection. However, there are only a few studies discussing the citation pattern of 'automation and control systems' subject, especially on its obsolescence characteristics. In this study, we identify the obsolescence patterns of this subject using the Laplace transfer-based citation model, with the aim to depict the phenomenon of 'hardness' or 'softness ${ }^{, 27}$ for the articles in this subject ${ }^{27}$.

Analysis of citation life cycles of the seminal journal articles is considered a valid approach in scientome$\operatorname{trics}^{28,29}$. A longitudinal analysis of the distribution of citations to these seminal works should reveal the growing, stable and declining research trends ${ }^{30}$. This study performs a ten-year longitudinal analysis on the obsolescence of 'automation and control systems' subject to examine its evolving research trend. Journals under the 'automation and control systems' section in Journal Citation Reports (JCR) (2008-17) were collected to perform this task. In order to clarify whether journals with different impact factors (IFs) could have different obsolescence characters, we collected journals that are divided into different journal impact factor (JIF) quartiles in each of the $J C R$ years (2008-2017).

The JIF quartile of $J C R$ is used for early rankings of science and social science journals, in the subject categories relevant to the journals based on their IF data. It is the quotient of a journal's rank in category $(X)$ and the total number of journals in the category $(Y)$, so that $(X / Y)=$ percentile rank $Z$. JIF quartile rankings are therefore derived for each journal in each of its subject categories according to the quartile of IF distribution which the journal occupies for that subject category. Q1 denotes the top $25 \%$ of IF distribution, Q2 the middle-high position (between top 50\% and top 25\%), Q3 the middle-low position (top 75\% to top 50\%) and Q4 the lowest position (bottom $25 \%$ of IF distribution).

We collected all the journals under each JIF quartile ranking (Q1-Q4) in each $J C R$ year from 2008 to 2017 in the 'automation and control systems' section. There are 72 journals indexed under this section by JCR in the ten years, with 27, 34, 33 and 30 journals in the JIF quartile ranking of Q1, Q2, Q3 and Q4 respectively. In order to eliminate the fluctuation of periodical membership to the JIF quartile ranking in different $J C R$ years and ensure the consistency of the experimental results, periodicals were sorted according to their frequency of occurrence in each quartile ranking. The periodicals that exist at least six times in one JIF quartile ranking in the ten years are regarded as the representatives of that quartile. Besides the limits on occurrence frequency, journals with incomplete citation data in JCR (2008-17) were also eliminated. Finally, we obtained a comprehensive consensus list of 40 journals. Table 1 shows the list journals classified 
RESEARCH ARTICLES

Table 1. Journals classification for the subject of 'automation and control systems'

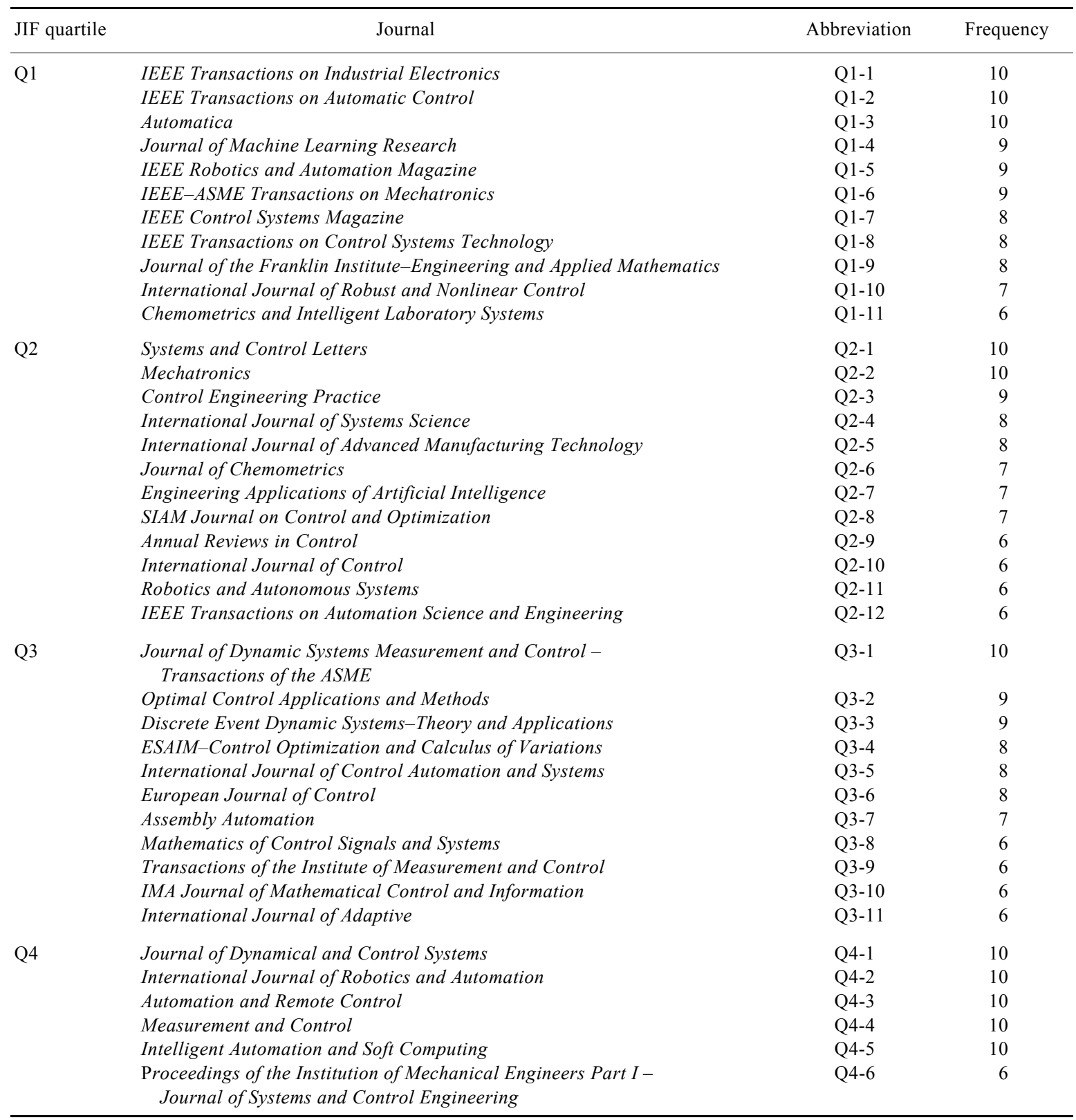

into Q1-Q4 under subject of 'automation and control systems' in $J C R$.

Then the 'cited journal data' for individual journals, as well as the 'cited category data' for 'automation and control systems' in the field level were directly retrieved from the JCR website (2008-17). Based on these citation data, the ageing time parameters were identified to make a longitude analysis of the obsolescence characteristics of this subject.

\section{Results and discussion}

Based on the 'cited category data' collected from JCR during 2008-2017 for 'automation and control systems', we calculated the cumulative citation rate distribution for this field in each collection year as shown in Table 2. $Y$ denotes the year in each row in the table, which is the $J C R$ year to collect the citation data. $Y-i$ is the $i$ th year before the collection year. Each row in Table 2 presents the cumulative citation rate distribution of articles in the subject 'automation and control systems' in the collection year.

Figure 3 shows the actual and fitted cumulative citation rate distribution curves for articles in 'automation and control systems'. Obviously, the actual cumulative citation rate distribution in curves each collection year are all well-fitted by the cumulative citation rate distribution function $C(T)$ in eq. (16). It indicates that the cumulative 
RESEARCH ARTICLES

Table 2. The cited data to articles in 'automation and control systems' from Journal Citation Reports (2008-2017)

\begin{tabular}{|c|c|c|c|c|c|c|c|c|c|c|c|}
\hline Year & $\mathrm{Y}$ & Y-1 & $\mathrm{Y}-2$ & $Y-3$ & $\mathrm{Y}-4$ & $Y-5$ & Y-6 & $Y-7$ & Y-8 & Y-9 & Others \\
\hline 2008 & 0.01 & 0.075 & 0.156 & 0.242 & 0.331 & 0.409 & 0.477 & 0.538 & 0.595 & 0.642 & 1 \\
\hline 2009 & 0.01 & 0.074 & 0.167 & 0.25 & 0.327 & 0.404 & 0.475 & 0.536 & 0.591 & 0.64 & 1 \\
\hline 2010 & 0.009 & 0.07 & 0.173 & 0.272 & 0.355 & 0.427 & 0.496 & 0.561 & 0.615 & 0.661 & 1 \\
\hline 2011 & 0.012 & 0.078 & 0.181 & 0.278 & 0.363 & 0.436 & 0.496 & 0.556 & 0.612 & 0.658 & 1 \\
\hline 2012 & 0.013 & 0.078 & 0.174 & 0.277 & 0.368 & 0.441 & 0.505 & 0.56 & 0.612 & 0.662 & 1 \\
\hline 2015 & 0.016 & 0.094 & 0.197 & 0.285 & 0.37 & 0.446 & 0.516 & 0.575 & 0.622 & 0.664 & 1 \\
\hline 2016 & 0.015 & 0.09 & 0.191 & 0.289 & 0.364 & 0.441 & 0.507 & 0.572 & 0.624 & 0.665 & 1 \\
\hline 2017 & 0.028 & 0.107 & 0.21 & 0.312 & 0.402 & 0.466 & 0.531 & 0.587 & 0.638 & 0.683 & 1 \\
\hline
\end{tabular}

$Y$ denotes the year in each row in Table 2, which is the JCR year to collect the citation data. $Y-i$ is the $i$ th year before the collection year.
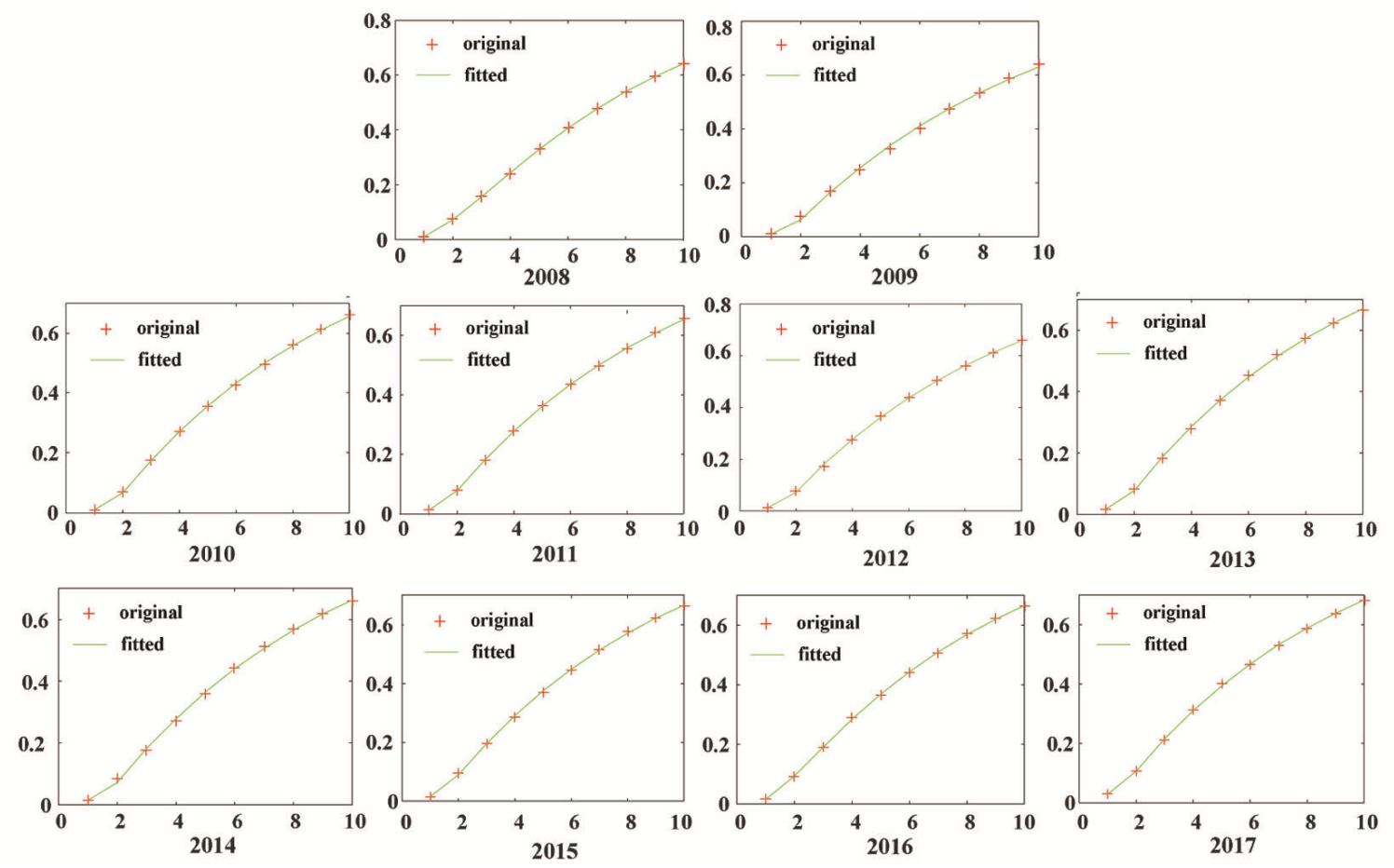

Figure 3. Actual and fitted cumulative citation distribution curves for 'automation and control systems'.

citation rate distribution function deduced by the Laplace transfer-based citation model is a good fit to the actual cumulative citation distribution.

In this way, the three time delay parameters $T_{1}, T_{s}$ and $\tau$ were deduced from eq. (16). The half-life of $T_{0.5}$ was calculated using eq. (18), once the three time parameters were obtained. Table 3 shows the identified time parameters for the field. The last column in the table gives the fitted error in each collection year. Obviously, the errors are considerably low, indicating that the time delay parameters calculated using the Laplace transfer-based citation model could well represent the actual obsolescence characteristics of articles in this subject.

Based on the identified ageing time parameters $T_{1}$ and $T_{0.5}$, Figure 4 further shows the evolving characters of obsolescence for the subject articles in the field level. Obviously, both $T_{1}$ and $T_{0.5}$ show a downward trend during the ten-year time period, indicating the accelerated trend for obsolescence of 'automation and control systems'. This reduced citing delay indicates that the published articles in this field have a faster knowledge diffusion process, which could be regarded as 'hardness', a concept used by Price to describe the phenomenon of shortened ageing of articles ${ }^{21}$. Figure 4 also shows that $T_{1}$ and $T_{0.5}$ express a different range of ageing values in each collection year, although they both have a downward ageing trend during the ten-year time period. As discussed above, $T_{1}$ could provide a more accurate value on the obsolescence character of articles than $T_{0.5}$, because the former considers all the citations. From the values of $T_{1}$ 


\section{RESEARCH ARTICLES}

in Table 3 and Figure 4, the average ageing rate of articles in 'automation and control systems' is 7-8 years, indicating that most citations would be given to the articles published 7-8 years before the collection year in the field.

We now depict the phenomenon of obsolescence at the journal level. The ageing characteristics of journals classified as different JIF quartiles are investigated to see if their obsolescence is still 'hardness', just as in the field level of 'automation and control systems'.

Following the same procedure, we calculated $T_{1}$ for each journal shown in Table 1 in each collection year and analysed the obsolescence characteristics of journals in different JIF quartiles. By taking year as the basic time unit, we analysed the evolving trend of $T_{1}$ with time. Figures 5-8 show the evolving properties of journal obsolescence.

Journals show diverse obsolescence properties regardless of their JIF quartiles. The value of $T_{1}$ changes in different years for the same journal, exhibiting different obsolescence trend (increasing or decreasing) of the journal. The obsolescence characters are also different in

Table 3. The identified time parameters for 'automation and control systems'

\begin{tabular}{llllll}
\hline Year & \multicolumn{1}{c}{$T_{1}$} & \multicolumn{1}{c}{$T_{\mathrm{s}}$} & \multicolumn{1}{c}{$\tau$} & \multicolumn{1}{c}{$T_{0.5}$} & \multicolumn{1}{c}{ Error } \\
\hline 2008 & 7.825 & 1.346 & 0.478 & 7.379 & 0 \\
2009 & 8.579 & 1.177 & 0.273 & 7.414 & 0.0005 \\
2010 & 7.966 & 1.18 & 0.294 & 7.058 & 0.0001 \\
2011 & 8.131 & 1.164 & 0.188 & 7.06 & 0 \\
2012 & 7.975 & 1.181 & 0.215 & 6.922 & 0.0001 \\
2013 & 7.783 & 1.186 & 0.186 & 6.708 & 0.0002 \\
2014 & 7.914 & 1.194 & 0.211 & 6.829 & 0.0004 \\
2015 & 7.991 & 1.135 & 0.113 & 6.771 & 0.0001 \\
2016 & 8.01 & 1.147 & 0.134 & 6.892 & 0.0001 \\
2017 & 7.729 & 1.087 & 0.041 & 6.527 & 0.0001 \\
\hline
\end{tabular}

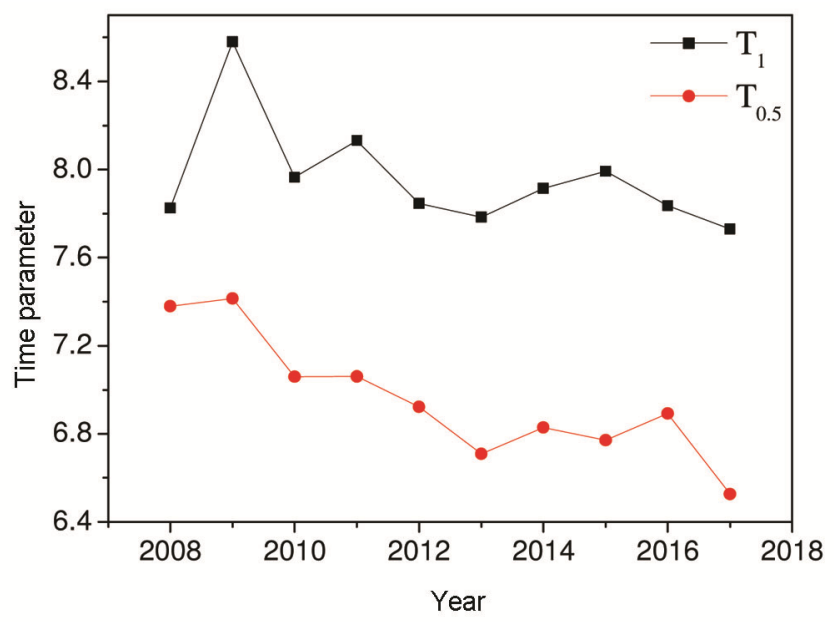

Figure 4. The evolving obsolescence characters for 'automation and control systems'. different JIF quartiles. There seems to be an extended trend in the ageing of journals from Q1 to Q4, because the upper limit of the ageing interval is about 15, 18, 35 and 20 years for periodicals in JIF quartiles Q1, Q2, Q3 and Q4 respectively. However, the upper limit of the

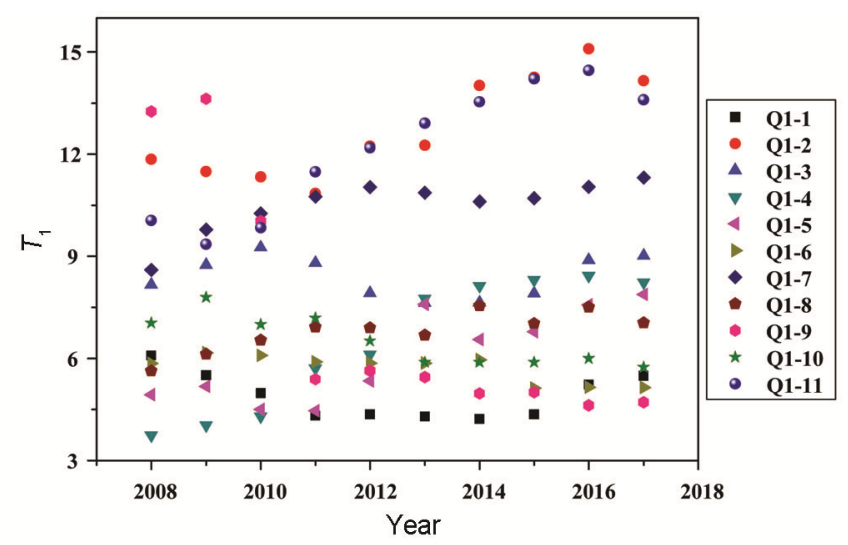

Figure 5. The evolving ageing characters for journals in Q1 in ten years $(2008-17)$

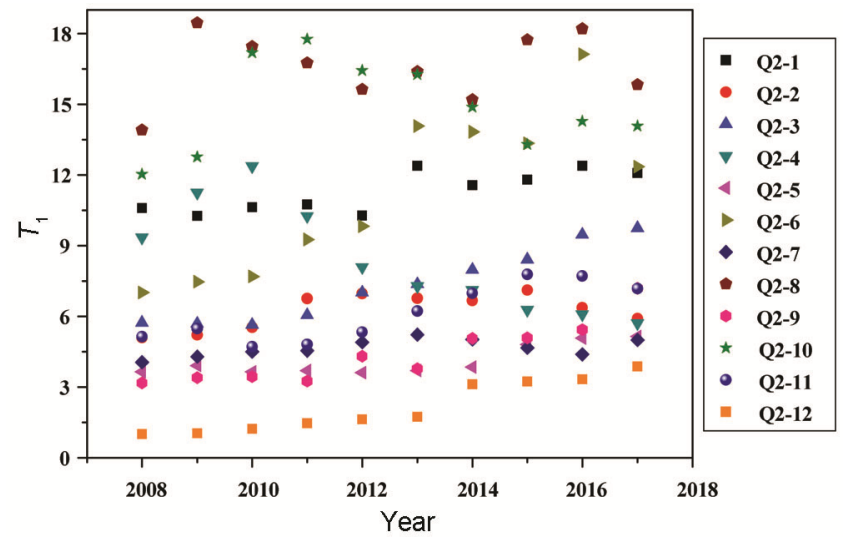

Figure 6. The evolving ageing characters for journals in $\mathrm{Q} 2$ in ten years $(2008-17)$.

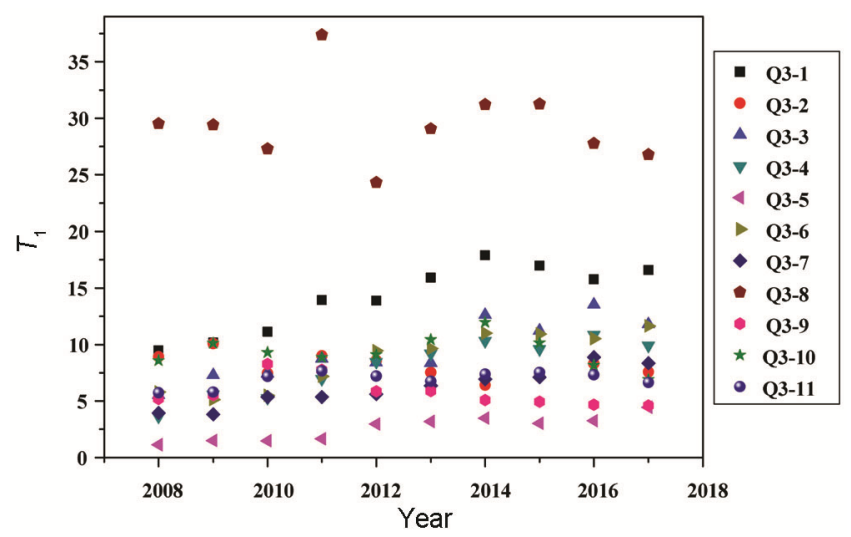

Figure 7. The evolving ageing characters for journals in Q3 in ten years $(2008-17)$.

CURRENT SCIENCE, VOL. 116, NO. 10, 25 MAY 2019 
Table 4. Number of journals with different ageing intervals in different Journal Impact Factor quartiles

\begin{tabular}{lccccc}
\hline Ageing interval (year) & Q1 & Q2 & Q3 & Q4 & Total \\
\hline $0-5$ & 1 & 2 & 1 & 0 & 4 \\
$5-10$ & 7 & 6 & 8 & 5 & 26 \\
$10-15$ & 3 & 3 & 1 & 0 & 7 \\
Beyond 15 & 0 & 1 & 1 & 1 & 3 \\
\hline
\end{tabular}

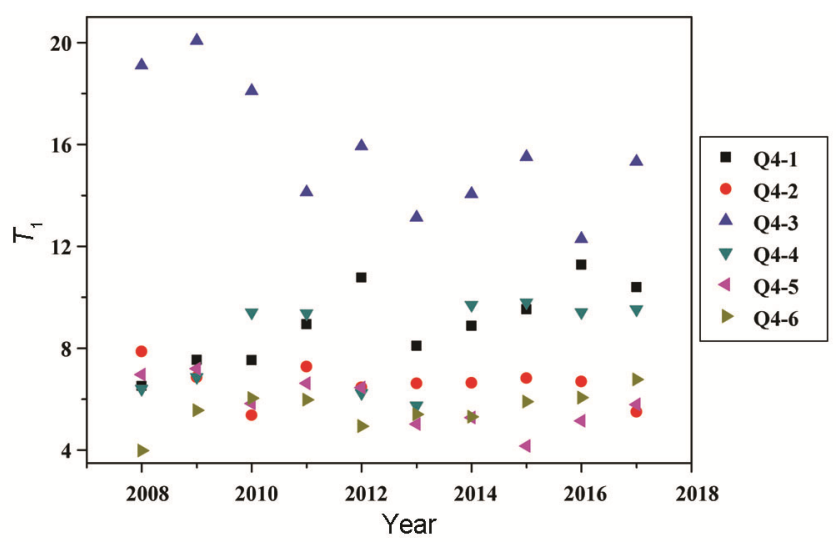

Figure 8. The evolving ageing characters for journals in Q4 in ten years $(2008-17)$.

ageing interval is affected by the ageing characteristics of very few periodicals, which cannot be regarded as an overall ageing law. From the distribution of the ageing intervals of periodicals in different JIF quartiles, we found that the ageing of most periodicals is within the range of 5-10 years. Table 4 shows the distribution of number of journals in each JIF quartile divided into different ageing intervals of $0-5,5-10,10-15$ and beyond 15 years, according to the value of $T_{1}$.

Obviously, the ageing of journals is mostly distributed in the range of 5-10 years, irrespective of the JIF quartile. There are 26 journals that meet this criterion which accounts for $65 \%$ of the total 40 journals shown in Table 1 . The result is consistent with the universal ageing interval of 'automation and control systems' in the field level. Also, $17.5 \%$ of the total number of periodicals were ageing in $10-15$ years, $10 \%$ in $0-5$ years, and only $7.5 \%$ ageing beyond 15 years.

Figures 5-8 show the evolving ageing characters of journals divided into different JIF quartiles. Among all the 40 journals considered here, 12 show a downward ageing trend in Figures 5-8, which coincides with the overall ageing tendency of the field. The articles in these journals would be ageing faster, showing the tendency of 'hardness'. However, the remaining 28 journals show an increasing ageing trend. The articles in these journals seem to become 'softer', with a lengthening tendency on their citation life. The results show that even in the same field, journals would exhibit different ageing characters.
It should be mentioned that although most journals (28 of 40), show an increasing ageing trend inconsistent with that at the field level, it is still a reasonable conclusion because only $55.5 \%(40 / 72)$ of the total 72 periodicals in subject were considered in this study for maintaining consistency of journals in different JIF quartiles. At the same time, different journals would have different number of publications, which cannot accurately represent the ageing trend of the discipline as a whole from the number of journals.

On the basis of the identified ageing parameter $T_{1}$, this study explored the relationship between ageing characteristics of periodicals and their impact factor (IF). Among the 40 journals analysed, only two show a decline in IF over the 10-year time period, while the remaining 38 journals show an increase in IF over the same period. It indicates that the ageing characteristics have no effect on IF of periodicals, because IF of most periodicals still shows an increasing trend regardless of whether the ageing is prolonged or shortened. Here, we further examine the relationship between the changing tendency of ageing parameter $T_{1}$ and IF of journals to explore whether there is any correlation between ageing and IF of journals. We calculated the increment of ageing parameter $T_{1}$ and IF in 10 years, which is expressed as $\Delta T_{1}$ and $\Delta \mathrm{IF}$ respectively

$$
\begin{aligned}
& \Delta T_{1}=T_{1(2017)}-T_{1(2008),} \\
& \Delta \mathrm{IF}_{1}=\mathrm{IF}_{(2017)}-\mathrm{IF}_{(2008)},
\end{aligned}
$$

Table 5 shows the relationship between $\Delta T_{1}$ and $\Delta \mathrm{IF}$ in different JIF quartiles; each value is the average under the conditions defined by rows and columns. For example, the value 0.649 in the first row is the mean of $\Delta T_{1}$ for journals in quartiles Q1. The value 2.612 in the first row is the mean value of $\Delta T_{1}$ for journals in quartiles Q1 with $\Delta T_{1}>0$. The value 1.45 of $\Delta \mathrm{IF}$ in the first row is the corresponding average increment of $\Delta \mathrm{IF}$ for these journals satisfying $\Delta T_{1}>0$ in quartile $\mathrm{Q} 1$. The results show that $\Delta T_{1}$ and $\Delta \mathrm{IF}$ both vary in different JIF quartiles. However, in general, compared with the periodicals in other JIF quartiles, the change interval of $\Delta T_{1}$ for the periodicals in Q1 is relatively small, which indicates the relative stable development status of these periodicals. At the same time, from the perspective of $\Delta I F$, the periodicals in Q1 have always maintained the fastest growth tendency 
Table 5. Relationship between $\Delta T_{1}$ and $\Delta \mathrm{IF}$ in different JIF quartiles

\begin{tabular}{lcccccc}
\hline Quartile & Average $\left(\Delta T_{1}\right)$ & Average $(\Delta \mathrm{IF})$ & Average $\left(\Delta T_{1}>0\right)$ & Average $(\Delta \mathrm{IF})$ & Average $\left(\Delta T_{1}<0\right)$ & Average $(\Delta \mathrm{IF})$ \\
\hline Q1 & 0.649 & 1.755 & 2.612 & 1.45 & -0.867 & 2.067 \\
Q2 & 1.944 & 1.055 & 2.451 & 1.009 & -3.635 & 1.551 \\
Q3 & 2.527 & 0.724 & 4.881 & 0.85 & -1.204 & 0.877 \\
Q4 & 0.414 & 0.464 & 3.269 & 0.459 & -2.441 \\
\hline
\end{tabular}

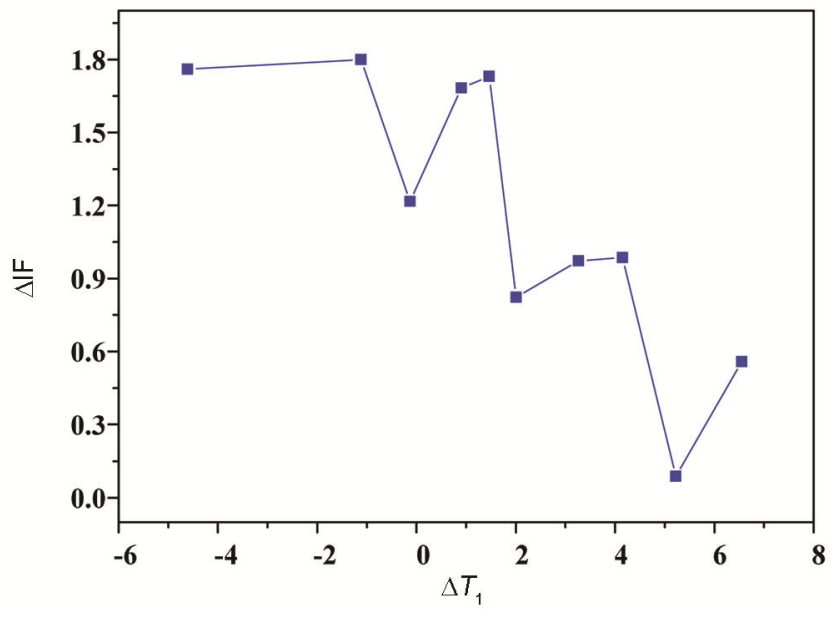

Figure 9. Relationship between $\Delta T_{1}$ and $\Delta \mathrm{IF}$ after four-point average.

in IF, regardless of whether the ageing trend is prolonged or shortened. Therefore, it seems that the change in JIF has no relation to its ageing trend, but only to the JIF quartiles of journals. The periodicals in Q1 have relatively fast growth trend of IF, followed by journals in Q2. And so on.

We deduced the relationship between $\Delta T_{1}$ and $\Delta \mathrm{IF}$, considering the change in $\Delta \mathrm{IF}$ as a function of $\Delta T_{1}$ for all the 40 journals. Figure 9 shows the relationship between $\Delta T_{1}$ and $\Delta \mathrm{IF}$. The data are smoothed by a four-point average. It shows that $\Delta \mathrm{IF}$ tends to decrease monotonously with increase of $\Delta T_{1}$. When the ageing trend exhibits a negative growth with $\Delta T_{1}<0$, the IF of periodicals increases rapidly; whereas when the ageing trend shows a positive growth with $\Delta T_{1}>0$, the IF of periodicals increases relatively slowly. The negative growth of the ageing trend corresponds to the acceleration of the obsolescence rate of journals, which indicates that the articles published in these journals will lose their value faster. Recent publications in these periodicals would win more citations, which enhances the growth of IF for the periodicals. On the contrary, the positive growth of ageing trend corresponds to the extension of the obsolescence rate. The results show that the value of articles published in these journals will decline at a slower rate, and these articles will have longer citation life than those published in journals with $\Delta T_{1}<0$. Readers will pay relatively more attention to the older articles in these periodicals compared with periodicals showing a short- ened ageing rate, which results in the slow increase of IF for these journals.

Therefore, although 'automation and control systems' exhibits a decreasing trend for obsolescence rate in the field level, journals in this field show different forms and more abundant obsolescence characteristics. These diverse changes in ageing characteristics affect the IF of journals. When the ageing trend reduces, periodicals will show an obvious growth trend of IF. On the contrary, when the ageing trend is prolonged, the growth rate of IF will be relatively slow.

\section{Conclusion}

Since the concept of obsolescence was first introduced to describe the phenomenon by which scientific publications are sparingly used over time, researchers have studied the ageing process of scientific literature and also the ageing characters in aggregated levels such as journals, subfields and fields. This study proposes a Laplace transfer-based citation model to identify the obsolescence time parameters expressed by the actual ageing process of articles. By taking the field of 'automation and control systems' as an example, a longitudinal obsolescence analysis of this field has been performed.

Experimental results show that the obsolescence time parameters identified in this study represent the obsolescence characteristics of this field. The subject 'automation and control systems' shows a moderate ageing rate and a shortened tendency in obsolescence, representing a phenomenon of 'hardness' as in the field level. However, journals exhibit diversified and more abundant ageing characteristics, of which 12 journals show accelerated ageing, while the other 28 journals prolong ageing. The relationship between $T_{1}$ and IF shows that the latter will increase irrespective of whether the ageing characteristics of journals is shortened or prolonged. There is a monotonic decreasing dependence between $\Delta \mathrm{IF}$ and $\Delta T_{1}$. When the ageing trend exhibits a negative growth with $\Delta T_{1}<0$, the IF of periodicals increases more significantly; whereas when the ageing trend shows a positive growth with $\Delta T_{1}>0$, IF of periodicals increases relatively slow.

1. Arao, L. H., Santos, M. J. V. D. and Guedes, V. L. D., The halflife and obsolescence of the literature science area: a contribution to the understanding the chronology of citations in academic activity. Qual. Quant. Meth. Lib., 2015, 603-610. 
2. Avramescu, A., Actuality and obsolescence of scientific literature. J. Am. Soc. Inf. Sci., 1979, 30, 296-303.

3. Line, M. B., The 'half-life' of periodical literature: apparent and real obsolescence. J. Doc., 1970, 26(1), 46-54.

4. Larivière, Archambault, V. É. and Gingras, Y., Long-term variations in the aging of scientific literature: from exponential growth to steady-state science (1900-2004). J. Am. Soc. Inf. Sci. Technol., 2008, 59(2), 288-296.

5. Nicholas, D., Huntington, P., Dobrowolski, T., Rowlands, I., Jamali, M., Hamid, R. and Polydoratou, P., Revisiting obsolescence and journal article decay. Inf. Proc. Manag., 2005, 41, 1441-1461.

6. Moed, H. F., Bibliometric measurement of research performance and Price's theory of differences among the sciences. Scientometrics, 1989, 15(5-6), 473-483.

7. Glänzel, W., Thijs, B. and Chi, P. S., The challenges to expand bibliometric studies from periodical literature to monographic literature with a new data source: The book citation index. Scientometrics, 2016, 109(3), 2165-2179.

8. Alvarado, R. U., Synchronic study of literature obsolescence: the case of Lotka's Law. Invest. Bibliotecol., 2014, 28(63), 85-113.

9. Onodera, N., Properties of an index of citation durability of an article. J. Informetr., 2016, 10(4), 981-1004.

10. Sotelo-Cruz, N., Atrian-Salazar, M. L. and Trujillo-Lopez, S., Indicators of obsolescence of the medical literature in a Mexican pediatric journal. Gaceta Med. Mexico, 2016, 152(2), 202-207.

11. Wang, M. Y., Li, S. and Chen, G. S., Detecting latent referential articles based on their vitality performance in the latest 2 years. Scientometrics, 2017, 112(3), 1557-1571.

12. Yin, Y. and Wang, D. S., The time dimension of science: connecting the past to the future. J. Informetr., 2017, 11(2), 608-621.

13. Glänzel, W. and Schoepflin, U., A bibliometric study on ageing and reception processes of scientific literature. J. Inf. Sci., 1995, 21(1), 37-53.

14. Glänzel, W. and Schoepflin, U., A bibliometric study of reference literature in the sciences and social sciences. Inf. Process. Manage., 1999, 35(1), 31-44.

15. Glänzel, W., Towards a model for diachronous and synchronous citation analyses. Scientometrics, 2004, 60(3), 511-522.

16. Bouabid, H. and Lariviere, V., The lengthening of papers' life expectancy: a diachronous analysis. Scientometrics, 2013, 97(3), 695-717.

17. Zhang, L. and Glänzel, W., A citation-based cross disciplinary study on literature aging: part I-the synchronous approach. Scientometrics, 2017, 111(3), 1573-1589.

18. Zhang, L. and Glänzel, W., A citation-based cross disciplinary study on literature aging: part II - diachronous aspects. Scientometrics, 2017, 111(3), 1559-1572.
19. Egghe, L., and Rousseau, R., The influence publication delays on the observed aging distribution of scientific literature. J. Am. Soc. Inf. Sci., 2000, 51, 158-165.

20. Yu, G., Yu, D. R. and Rong, Y. H., The mathematical models of the periodical literature publishing process. Inf. Process. Manage., 2000, 36, 401-414.

21. Yu, G., Rong, Y. H. and Li, Y. J., Mathematical model of delay in the secondary literature publishing process. Scientometrics, 2003, 58(3), 773-685.

22. Yu, G., Yu, D. R. and Li, Y. J., The universal expression of periodical average publication delay at steady state. Scientometrics, 2004, 60(2), 121-129.

23. Yu, G. and Li, Y. J., Parameter identification of the observed citation distribution. Scientometrics, 2007, 71(2), 339-348.

24. Ali, S., Aslam, M. and Kazml, S. M. A., Heterogeneous data analysis using a mixture of Laplace models with conjugate priors. Int. J. Syst. Sci., 2014, 45(12), 2619-2636.

25. Kocabas, I., Application of iterated Laplace transformation to tracer transients in heterogeneous porous media. J. Franklin Inst. - Eng. Appl. Math., 2011, 348(7), 1339-1362.

26. Sheng, H., Li., Y. and Chen, Y. Q., Application of numerical inverse Laplace transform algorithms in fractional calculus. J. Franklin Inst. - Eng. Appl. Math., 2011, 348(2), 315-330.

27. Price, D. J. D., Citation measures of hard science, soft science, technology, and nonscience. In Communication among Scientists and Engineers, Heath Lexington, MA, USA, 1970, pp. 3-22.

28. Calabretta, G., Durisin, B. and Ogliengo, M., Uncovering the intellectual structure of research in business ethics: a journey through the history, the classics, and the pillars of journal of business ethics. J. Business Ethics, 2011, 104(4), 499-524.

29. Ho, Y. S., A bibliometric analysis of highly cited articles in materials science. Curr. Sci., 2014, 107(9), 1565-1572.

30. Higham, K. W., Governale, M., Jaffe, A. B. and Zulicke, U., Unraveling the dynamics of growth, aging and inflation for citations to scientific articles from specific research fields. J. Informetr., 2017, 11(4), 1190-1200.

ACKNOWLEDGEMENTS. This work was supported by the National Natural Science Foundation of China (Grant Nos 71473034; 717D1063; 61806049), and the financial assistance from Postdoctoral Scientific Research Developmental Fund of Heilongjiang Province (Grant No. LBH-Q16003).

Received 29 November 2018; revised accepted 11 February 2019 doi: $10.18520 / \mathrm{cs} / \mathrm{v} 116 / \mathrm{i} 10 / 1696-1705$ 